\title{
Learning in Robotics
}

\author{
Amir Mosavi \\ Kalman Kando Faculty of \\ Electrical Engineering, \\ Obuda University, Hungary
}

\author{
Annamaria Varkonyi \\ Kalman Kando Faculty of \\ Electrical Engineering, \\ Obuda University, Hungary
}

\begin{abstract}
Machine learning is currently identified as one of the major parts of the research in Robotics. However the advanced concept of machine learning plus optimization reported effective for developing learning systems. This article considers the novel integration of machine learning and optimization for the complex and dynamic context of Robot learning. Further the proposed case study presents an effective framework for learning and solving the global optimization problem within the context of Robotics and learning.
\end{abstract}

\section{Keywords}

Predictive analytics, machine learning, optimization, robotics

\section{INTRODUCTION}

Learning is a major part of the research in Robotics [1]. Machine learning algorithms in robotics in particular, are being used to tackle learning tasks where large quantities of datasets are available which enable Robots to effectively teach themselves accordingly [2]. Yet application of machine learning in Robotics which highly contributes to Robot learning is vast and yet progressing in a fast pace [18]. Robot vision [3], Robot navigation [4], field Robotics [5], humanoid Robotics [6], legged locomotion [13], off-road rough-terrain mobile Robot navigation [14], modeling vehicle dynamics [15], medical and surgery Robotics [15], are few of the areas within Robotics for which utilizing machine learning technologies has become popular. It is, therefore clearly evidenced that machine learning has in recent years become an essential part of Robotics. And this has been in fact a response to the frustration with the problems for which it has been proven difficult to conventional coding solutions. For instance in a variety of Robotics platforms such as humanoid robotics [12], and legged locomotion [13], the imitation learning techniques [16], and inverse optimal control methods [17] play an increasingly important role. In such areas, for instance, the alternative approach of programming-bydemonstration [18] is utilized where Robot behaviors are created by involvement of expert demonstration. In addition the supervised learning techniques [19] have become norm within field robotics in rough terrain. Furthermore selfsupervised learning [20] is applied to generate training examples for self-improvement enabling Robots to effectively teach themselves. A number of the most notable machine learning technologies utilized in Robotics realm includes; reinforcement learning [21], supervised learning [19], selfsupervised learning [20], multi-agent learning [22], autonomous science [23], machine learning techniques for big data [24], imitation learning techniques [16], Robot programming by demonstration [25], and multi-agent learning [26].

\section{LEARNING AND OPTIMIZATION IN ROBOTICS}

Machine learning as a sub-field of computer science has evolved from the study of pattern recognition and computational learning theory [7]. Machine learning is considered as a field of study in artificial intelligence that gives computers the ability to learn from data [8]. To do so machine learning explores the development of models that can predict and learn from an available dataset [9]. Such models operate with the aid of algorithms capable of making datadriven predictions rather than following explicit codes [10]. Consequently machine learning is often used in a range of problems where designing precise algorithms is not practical. In this sense machine learning can replace the human expertise in information treatment [11]. To doing so machine learning provides the algorithmic tools for dealing with datasets and providing predictions. In fact machine learning tends to imitate human skills, which in most cases, act exceptional in identifying satisfactory solutions by theoretical or experience-based considerations [12].

The intersection research area of optimization and machine learning has recently engaged leading scientists [27]. Machine learning has made benefit from optimization and on the other hand machine learning contributed to optimization as well. Today machine learning is seen as an exceptional replacement for human expertise in information manipulation [28]. In addition machine learning has the proven ability to simplify optimization functions [29]. Optimization on the other hand is the source of immense power for automatically improving decisions [30]. However in real-life applications, including Robotics, optimization has not had the chance to be used to its full potential [31]. This has been often due to the absence, complexity, or inefficient optimization functions of the complicated problem at hand [34]. Yet in such cases machine learning has shown the ability of modeling whole or part of the optimization functions on the basis of the availability of a reliable dataset [32]. A number of case studies concerning Robotics problems have been surveyed in literature, e.g [33], where machine learning technologies simplify complicated optimization functions.

Nevertheless the long-term vision for Robot learning would be the development of a fully automated system with selfservice usage [18]. To reach this goal the novel idea of integration of machine learning and optimization [28] aims at simplifying the whole learning process by automating the decision-making tasks in an effective manner without requiring a costly learning curve for the final-user [7]. In this context the learning process is seen as a byproduct of an automated optimal decision.

Learning from the available dataset integrated with optimization can be applied to a wide range of complex, dynamic, and stochastic problems [27]. Such integration has been reported exceptional in increasing the automation level by putting more power at the hands of final-user [34]. Finaluser should however specify dataset, desired outputs and CPU time. CPU time is to be set to put a limitation on optimization algorithms' run-time which can be referred as "learning time". 
The novel integration of machine learning and optimization has already been used in solving numerous complex cases [39]. Decision-making in complex geometrical problems [35], patient's diagnosis problem and healthcare decision-making [36], multiobjective optimization problems [37] wireless access point optimization [38], mobile Robot navigation [39], business intelligence and business decision-making models [40], automated decision-making [41] bioinformatics and big data [29] are few examples. Considering these examples, it is observed that once a combination of right machine learning technologies and optimization algorithms designed, suitable for the problem at hand, further algorithm selection, adaptation, and integration, are done in an automated way, and a complete solution for learning is delivered to the final user. As the result the real-life decision-making tasks with the diverse, stochastic, and dynamic nature can be handled smoothly and continuously. Within the framework of such integration, it is expected that algorithm selection and adaptation are done in an automated way, and a comprehensive solution is delivered to the final user.

\subsection{Integration of Learning and Optimization}

Depending on the characteristics of the problem at hand and availability of dataset an arrangement of local and/or global optimization algorithms [42] is essential to come up with an optimal decision. Yet local searches leading to locally optimum is an essential principle for solving the discrete and continuous optimization problems. In this context designing a system that is capable of curing local optimum traps is desirable. In order to cure the local optimum traps in an automated manner reactive search optimization (RSO) [32] is used.

RSO methodology implements an integration of machine learning techniques into local and heuristics search [29] for solving real-life optimization problems [5]. RSO includes a so-called "machine learning application builder" [39] employed to design a system which receives dataset, guide the research, and delivers a competitive application. In fact the "machine learning application builder" imitates the human skill in providing the automation to the system which is responsible for algorithm selection and parameter tuning. In fact human brain quickly learns and drives future decisions based on previous observations [8]. This is the main inspiration source for inserting machine learning techniques into the learning curve. This is referred as brain-computer optimization (BCO) [43] which is an important building block of RSO. Building blocks of RSO neural networks, statistics, artificial intelligence, reinforcement learning, and active or query learning [28]. Characteristics of RSO include learning on the job, rapid generation and analysis of many alternatives, efficient analysis of what-if scenarios, flexible decision support, diversity of solutions and anytime solutions [29].

\section{ALGORITHM IN ACTION}

The proposed case study aims at evaluating the RSO methodology with the aid of the educational Robotics kit of LEGO Mindstorms.

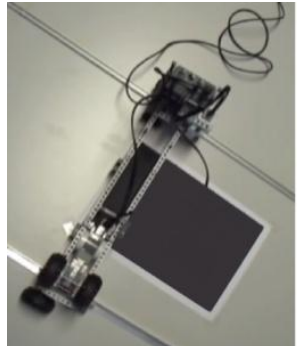

a)

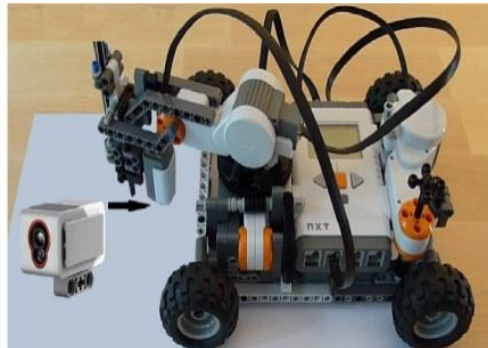

b)
Fig. 1: (a) Sample robot arrangement (b) Standard LEGO Mindstorms kit

The objective is to evaluate the ability of learning of a mobile Robot in locating the darkest spot of a paper sheet (figure 1a). The number of the light intensity inspections is limited to a total of nine sample points within the Cartesian coordinate.

\subsection{LEGO Mindstorms robot}

Massachusetts Institute of Technology collaborated with LEGO group in developing the Programmable Brick of LEGO Mindstorm robot in 1988 [44]. Since then Robot programming was introduced to the young generation as an exciting and entertaining task. Later on world leading robotics research and education centers such as Carnegie Mellon's Robotics Institute, University of Iowa, and Columbia University recognized LEGO Mindstorm robot as an educational robotics kit. Today numerous universities around the world teach artificial intelligence classes with the aid of LEGO Mindstorms platform and many literatures describe the educational benefits of this practice [45]. Generally LEGO Mindstorms platform is introduced as a low cost and easy to use device for the educational purposes where students can practice basic image processing and motion control in addition to transferring their artificial intelligence knowledge to the development of control programs [44].

\subsection{Implementation}

In order to move in a controlled manner within the Cartesian coordinate system in the identified territory the Robot has been upgraded to a new arrangement (figure $2 b$ ). There are around 200 LEGO parts coming as the standard LEGO Mindstorms kit to build a Robot [45]. In the presented case study a simple arrangement provides the limited straight movements of the mobile Robot. With adding a Matrix kit to a conventional matrix building system a x-y table is created. In addition the mobile Robot is equipped with a color sensor which measures the light intensity (figure $2 b$ ).
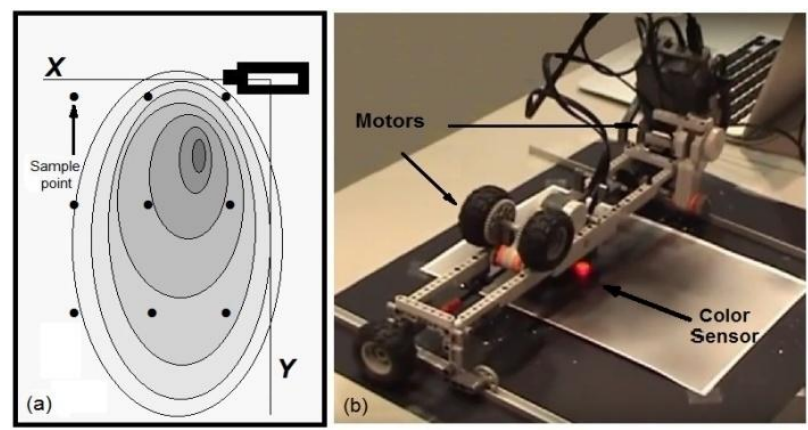

Fig 2: Robot arrangement; (a) Black and white paper sheet presenting a random intensity of light presented over a Cartesian coordinate system with the coordinates of nine sample points (b) Robot in action; arrangement of LEGO 


\section{Mindstorms Robot equipped with a color sensor and a Matrix kits}

The The prospector here is a color sensor which measures the light intensity and reports to a simple code via a USB cable configuration. According to the simple code as the Robot moves along the Cartesian coordinate over the black and white paper nine samples of light intensity are taken. Then to connect this external code to the Robot learning system the measured points are connected to a design of experiment
(DOE) [46] module to further import into a function generator. In this stage we can plot the results on a 3D graph as it is presented in the figure 2a. A second degree Polynomial fit estimates the distribution of the nine sample points. And RSO runs a continues optimizer in order to predict the optimal points and generate the optimum (figure $3 b$ ). Robot then is directed to the newly generated optimum accordingly. Matching the predicted optimum with the darkest spot of the sheet proves the accuracy of the model (figure $3 \mathrm{c}$ ).

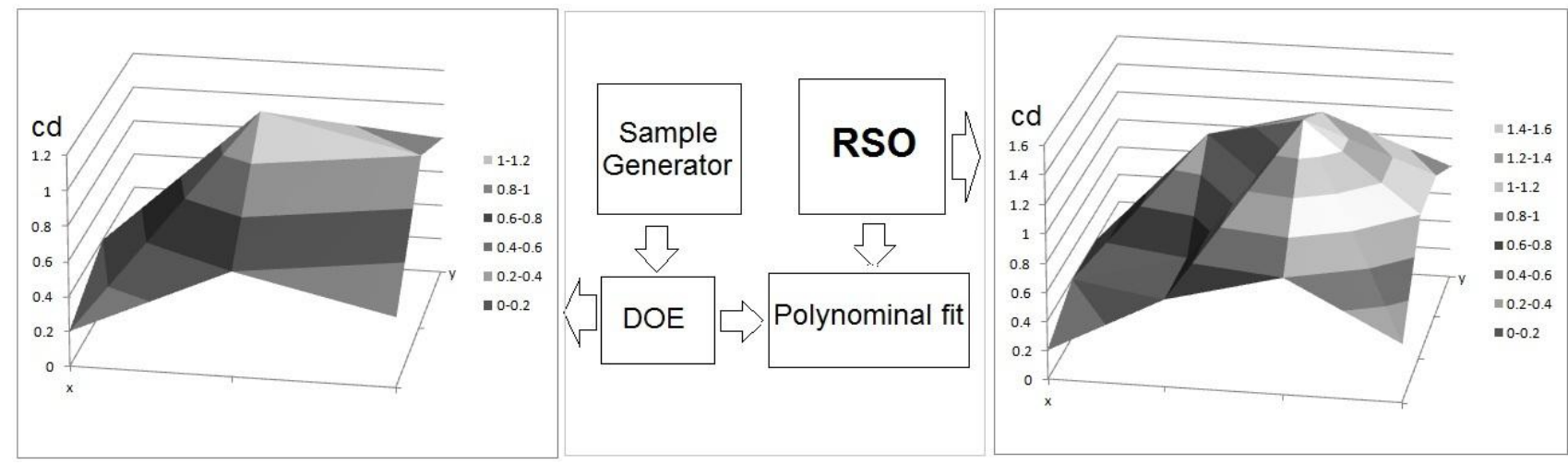

Fig. 3. (a) 3D graph of the points primary been measured (b) Building blocks of RSO methodology of learning (c) 3D graph of the newly generated points

\section{CONCLUSIONS}

Machine learning is one of the major parts of the research in Robotics. Yet the advanced concept of machine learning plus optimization has been recently reported effective for developing learning systems. The paper considers the novel integration of machine learning and optimization for the complex and dynamic context of Robot learning. RSO is introduced as a methodology to implement an integration of machine learning techniques into local and heuristics optimization for Robot learning. In the proposed case study RSO presents an effective framework for learning and solving the global optimization problem. In the case study the ability of learning of a mobile Robot in locating the darkest spot of a paper sheet is evaluated. Matching the predicted optimum with the darkest spot of the sheet proves the accuracy of the model.

\section{REFERENCES}

[1] Connell, J.H., Mahadevan, S.: editors. Robot learning. Springer Science \& Business Media (2012)

[2] Knox, W.B., Glass, B.D., Love, B.C., Maddox, W.T., Stone, P.: How humans teach agents. International Journal of Social Robotics 4(4):409-21 (2012)

[3] Rosten, E., Drummond, T.: Machine learning for highspeed corner detection. In Computer Vision-ECCV. Springer Berlin Heidelberg 430-443 (2006)

[4] Sofman, B., Lin, E., Bagnell, J.A., Cole, J., Vandapel., N., Stentz, A.: Improving robot navigation through self-supervised online learning. Journal of Field Robotics. 23, 59-75. (2006)

[5] Yang, S.Y., Jin, S.M., Kwon, S.K.: Remote control system of industrial field robot. InIndustrial Informatics, 6th IEEE International Conference on, 442-447 (2008)

[6] Peters, J., Vijayakumar, S., Schaal, S.: Reinforcement learning for humanoid robotics. In Proceedings of the third IEEE-RAS international conference on humanoid robots 1-20 (2003)
[7] Bishop, CM., Nasrabadi, NM.: Pattern Recognition and Machine Learning. Journal of Electronic Imaging 16(4), (2007)

[8] Michalski, R.S., Carbonell, JG., Mitchell, TM.: editors. Machine learning: An artificial intelligence approach. Springer Science \& Business Media (2013)

[9] Han, J., Kamber, M., Pei, J.: Data mining: concepts and techniques. Elsevier (2011)

[10] Waller, M.A., Fawcett, SE.: Data science, predictive analytics, and big data: a revolution that will transform supply chain design and management. Journal of Business Logistics 34(2):77-84 (2013)

[11] Battiti. R., Brunato. M.: Reactive search optimization: learning while optimizing. InHandbook of Metaheuristics. Springer US. 543-571 (2010)

[12] Murphy, R.R.: Human-robot interaction in rescue robotics. Systems, Man, and Cybernetics, Part C: Applications and Reviews, IEEE Transactions on. 34(2) 138-53 (2004)

[13] Kohl N, Stone P.: Machine learning for fast quadrupedal locomotion. InAAAI 611-616 (2004)

[14] Popp, K., Schiehlen, W.: Ground vehicle dynamics. Springer Berlin Heidelberg; 2010.

[15] Taylor, R.H., Menciassi, A., Fichtinger, G., Dario, P., Medical robotics and computer-integrated surgery. InSpringer handbook of robotics, Springer Berlin Heidelberg 1199-1222 (2008)

[16] Nehaniv, C.L., Dautenhahn, K.: editors. Imitation and social learning in robots, humans and animals: behavioural, social and communicative dimensions. Cambridge University Press (2007)

[17] Mombaur, K., Truong, A., Laumond, J.P.: From human to humanoid locomotion-an inverse optimal control approach. Autonomous robots. 28(3) 369-83 (2010) 
[18] Argall, B.D., Chernova, S., Veloso, M., Browning, B.: A survey of robot learning from demonstration. Robotics and autonomous systems. 57(5) 469-83 (2009)

[19] Mozos, O.M., Stachniss, C., Burgard, W.: Supervised learning of places from range data using adaboost. In Robotics and Automation, Proceedings of the 2005 IEEE International Conference on 1730-1735 (2005)

[20] Stavens, D., Thrun, S. A.: self-supervised terrain roughness estimator for off-road autonomous driving. arXiv preprint arXiv:1206.6872 (2012)

[21] Kober, J., Bagnell, J.A., Peters, J.: Reinforcement learning in robotics: A survey. International Journal of Robotics Research. 32(11) 1238-74 (2005)

[22] Kalra, N., Zlot, R., Dias, M.B., Stentz, A.: Market-Based Multirobot Coordination: A Comprehensive Survey and Analysis. Report Carnegie Mellon University (2005)

[23] Hebert, MH., Thorpe, C.E., Stentz, A.: editors. Intelligent unmanned ground vehicles: autonomous navigation research at Carnegie Mellon. Springer Science \& Business Media (2012)

[24] Wing, J.M.: Computational thinking. Communications of the ACM 49(3) (2006)

[25] Billard, A., Calinon, S., Dillmann, R., Schaal, S.: Robot programming by demonstration. InSpringer handbook of robotics Springer Berlin Heidelberg 1371-1394 (2008)

[26] Panait, L., Luke, S.: Cooperative multi-agent learning: The state of the art. Autonomous Agents and MultiAgent Systems 11(3) 387-434 (2005)

[27] Sra, S., Nowozin, S., Wright, S.J.: Optimization for machine learning. Mit Press (2012)

[28] Battiti, R., Brunato, M., Mascia, F.: Reactive search and intelligent optimization. Springer Science \& Business Media (2008)

[29] Amir Mosavi, Annamaria Varkonyi, Integration of Machine Learning and Optimization for Robot Learning, Advances in Intelligent Systems and Computing, Springer-Verlag Berlin Heidelberg (2016)

[30] Shim, J.P., Warkentin, M., Courtney, J.F., Power D.J, Sharda, R., Carlsson, C.: Past, present, and future of decision support technology. Decision support systems 33(2) 111-126 (2002)

[31] Toussaint, M., Ritter, H., Brock, O.: The Optimization Route to Robotics-and Alternatives. KI-Künstliche Intelligenz 29(4) 379-88 (2015)

[32] Mosavi, Amir, and Annamaria Varkonyi, Machine learning for artificial intelligence and Robotics, International Journal of Applied Mathematics, Electronics and Computers, (2016)
[33] Stone, P., Veloso, M.: Multiagent systems: A survey from a machine learning perspective. Autonomous Robots. 8(3) 345-83 (2000)

[34] Battiti, R., Brunato, M.: The LION way. Machine Learning Plus Intelligent Optimization. Applied Simulation and Modelling (2013)

[35] Mosavi, A.: Decision-Making in Complicated Geometrical Problems. International Journal of Computer Applications 87(19) (2014)

[36] Brunato M, Battiti R. Learning and intelligent optimization (LION): one ring to rule them all. Proceedings of the VLDB Endowment 6(11) 1176-7 (2013)

[37] Mosavi, A., Vaezipour, A.: Reactive Search Optimization; Application to Multiobjective Optimization Problems. Applied Mathematics 1572-82 (2012)

[38] Battiti, R., Brunato, M., Delai, A.: Optimal Wireless Access Point Placement for Location-Dependent Services. Technical Report \# DIT-03-052, University of Trento, Italy (2010)

[39] Battiti, R., Brunato, M.: The LION Way: Machine Learning plus Intelligent Optimization. Trento University, LIONlab (2014)

[40] Battiti, R., Brunato, M.: Reactive Business Intelligence. From Data to Models to Insight, Reactive Search Srl, Italy. (2011)

[41] Mosavi, A., Varkonyi-Koczy, A., Fullsack, M.: Combination of Machine Learning and Optimization for Automated Decision-Making. In: Conference on Multiple Criteria Decision Making MCDM, Hamburg, Germany (2015)

[42] Horst, R., Pardalos, P.M.: editors. Handbook of global optimization. Springer Science \& Business Media (2013)

[43] Battiti, R., Passerini, A.: Brain-computer evolutionary multiobjective optimization: a genetic algorithm adapting to the decision maker. Evolutionary Computation, IEEE Transactions on. 14(5) 671-87 (2010)

[44] Kim, S.H., Jeon. J.W.: Introduction for Freshmen to Embedded Systems Using LEGO Mindstorms. IEEE Transactions on Education. 52(1) 99-108 (2009)

[45] Parsons, S., Sklar, E.: Teaching AI using LEGO mindstorms. In AAAI Spring Symposium (2004)

[46] Goupy, J., Creighton, L.: Introduction to design of experiments with JMP examples. SAS Publishing (2007)

[47] Mosavi, Amir, and Annamaria R. Varkonyi-Koczy. Integration of Machine Learning and Optimization for Robot Learning. In Recent Global Research and Education: Technological Challenges, pp. 349-355. Springer International Publishing, (2017) 